la revue La revue pour l'histoire du CNRS

POUR LHISTORE Du CNRS $\quad 5 \mid 2001$

Des laboratoires à l'étranger

\title{
La sauvegarde du patrimoine instrumental
}

Conférence-débat du 6 juin 2001

(2) OpenEdition

Journals

Édition électronique

URL : https://journals.openedition.org/histoire-cnrs/3382

DOI : 10.4000/histoire-cnrs.3382

ISSN : 1955-2408

Éditeur

CNRS Éditions

Édition imprimée

Date de publication : 5 novembre 2001

ISBN : 978-2-271-05925-3

ISSN : $1298-9800$

Référence électronique

«La sauvegarde du patrimoine instrumental », La revue pour l'histoire du CNRS [En ligne], 5 | 2001, mis en ligne le 20 juin 2007, consulté le 20 mai 2021. URL : http://journals.openedition.org/histoire-cnrs/ 3382 ; DOI : https://doi.org/10.4000/histoire-cnrs.3382

Ce document a été généré automatiquement le 20 mai 2021.

Comité pour l'histoire du CNRS 


\section{La sauvegarde du patrimoine instrumental}

Conférence-débat du 6 juin 2001

\section{NOTE DE L'ÉDITEUR}

Les auteurs Pierre Chavel, Françoise Allain et Gérard Roger remercient leurs nombreux collègues qui contribuent à la mise en place du « mini-musée » de l'Institut d'optique. Serge Equilbey est d'une grande aide pour la constitution d'une documentation iconographique.

L'instrumentation scientifique : un élément essentiel du patrimoine (A)

1 Le Comité pour l'histoire du CNRS a décidé de se préoccuper de la sauvegarde du patrimoine instrumental qui a contribué, au fil du temps, à l'édification des connaissances scientifiques dans nos laboratoires. On ne peut que s'en réjouir car ce patrimoine n'est généralement pas considéré comme faisant partie d'un patrimoine culturel national, et ce y compris à l'intérieur même des laboratoires et malgré l'existence de musées scientifiques remarquables.

2 On pourrait peut-être avancer deux explications à cela. D'une part, le fait que la culture scientifique n' est souvent pas considérée comme faisant partie de «la culture » tout court, au même titre que les arts et les lettres, par exemple. D'autre part, les scientifiques eux-mêmes sont davantage sensibles à l'évolution et à l'amélioration des techniques instrumentales qu'à la conservation de «vieilles machines » du passé sans intérêt immédiat pour eux. Dit d'une autre manière, l'évolution scientifique est vue, surtout par les scientifiques, comme projetée dans le temps, les objets du passé perdant de l'importance au fur et à mesure que les connaissances progressent.

3 Il faut donc souvent attendre de longues années pour que, dans un souci de reconstitution historique, on s'occupe de l'appareillage qui a déterminé telle ou telle avancée scientifique particulièrement importante. Et, souvent, il est alors trop tard tant 
pour reconstituer des appareils qui entre-temps ont disparu ou, dans le meilleur des cas, ont été profondément transformés, que pour recueillir les témoignages des acteurs d'événements scientifiques importants.

Une fois l'importance du patrimoine scientifique reconnue, encore faut-il déterminer la meilleure manière d'agir pour que la sauvegarde ne se réduise pas à une accumulation disparate d'objets ou à des catalogues plus ou moins exhaustifs du matériel existant dans les laboratoires. De même qu un musée d'art doit être organisé, la conservation du patrimoine instrumental scientifique doit être guidée par quelques idées bien définies et être intégrée à un dessein.

Dans la démarche de sauvegarde du patrimoine instrumental on pourra mettre en valeur, par exemple, les prototypes qui, après avoir connu une évolution continue dans les laboratoires, sont devenus des appareillages commercialisés et utilisés quotidiennement dans de nombreux endroits du monde. Il serait intéressant de disposer de l'appareil initial, tel qu'il était au moment où a été mise en oeuvre l'idée clé qui a justifié son succès postérieur. Or, cela n' est pas toujours facile sans l'aide des personnes qui ont participé à cette mise en œuvre.

On pourra s intéresser aussi particulièrement aux grands équipements dans la mesure où ils touchent beaucoup de monde dans la communauté scientifique, notamment dans les domaines de la physique et de l'astrophysique. Les synchrotrons, les réacteurs nucléaires, les accélérateurs de particules, ont l'avantage de ne pas poser le problème de la pertinence du choix des objets à conserver. En effet, en moyenne, les progrès et les innovations instrumentales y sont mieux identifiés. Cela est dû au fait que, étant donné le volume des investissements nécessaires, l'évolution se fait, le plus souvent, d'une manière discontinue. Mais ce sont alors les coûts d'entretien du matériel conservé qui peuvent s' avérer prohibitifs.

7 Il est certain que, si l'on veut réellement agir en vue de la sauvegarde du patrimoine instrumental, de nombreux problèmes seront à résoudre : le choix des espaces, des lieux, l'entretien de ces matériels, leur maintien en état de marche, l'établissement de critères de sélection. On ne peut tout conserver, même si c' est une tendance actuelle, curieusement en contradiction avec la faible fiabilité des moyens de conservation. Du reste, la communauté scientifique, toujours tournée vers l'avenir, ne verrait pas forcément d'un bon oeil que trop de crédits soient consacrés à ce domaine, au détriment de la recherche elle-même.

8 La conservation du patrimoine peut être assurée, bien sûr, et à moindres frais, par des documents audiovisuels. Cependant, ces documents, bien que nécessaires, ne sauraient remplacer la possibilité d'approche et d'étude des appareils eux-mêmes. Ils ne peuvent être qu un complément, un "plus ", permettant de toucher le plus grand nombre possible de personnes intéressées. Ils sont utiles aussi pour établir une évolution historique ou pour souligner des liens interdisciplinaires.

Quel est donc le créneau dévolu à la sauvegarde des matériels scientifiques? Comme souvent, il est plus facile de délimiter le domaine d'une manière négative. Il ne s'agit donc ni de créer des musées ni d'entasser tout le matériel hors d'usage des laboratoires. En fait, à mon sens, ce serait aux laboratoires d'établir des "parcours scientifiques", 
comme cela existe déjà dans certaines universités et centres de recherche, de les rendre vivants et de leur faire jouer un rôle pédagogique, en priorité auprès des étudiants. Aussi, la pluridisciplinarité devrait être privilégiée dans ces parcours, faisant comprendre que tel ou tel appareil est, en fait, le résultat de la confluence à un moment et dans un laboratoire donnés de plusieurs apports provenant souvent de domaines lointains.

Les sociétés savantes, où se croisent naturellement des scientifiques d'origines diverses et presque toujours intéressés par l'histoire des sciences, devraient aussi être mises à contribution pour la définition et la réalisation de parcours scientifiques bien documentés et attirants. Certaines de ces sociétés se sont préoccupées de la place de la science dans l'histoire de la culture et ont des actions concrètes, telles que la récupération de cahiers et $\mathrm{d}$ 'appareillages scientifiques anciens dans quelques laboratoires.

11 Enfin, il faut espérer que la mise en oeuvre de la sauvegarde du patrimoine instrumental constitue une contribution importante au rapprochement, voire à l'intégration, de la culture scientifique dans une sphère culturelle qui la considère, souvent à tort, comme oublieuse de son propre passé. Les conférences-débats organisées par le Comité pour l'histoire du CNRS devraient permettre de motiver les collègues scientifiques, acteurs actuels des progrès techniques, et aussi d'attirer l'attention des décideurs.

Le patrimoine instrumental en neurosciences vu par un électrophysiologiste

(B)Introduction

12 Au cours du demi-siècle d'existence du CNRS, les méthodes mises en oeuvre dans les neurosciences ont fait des progrès spectaculaires liés évidemment à l'évolution des idées mais aussi aux progrès de l'instrumentation. Cela peut être illustré par quelques exemples pris en électrophysiologie, que l'on se réfère aux systèmes d'observation, aux appareils de micro-positionnement, aux appareils de mesure.

Le poste d'électrophysiologie

13 Un poste de travail électrophysiologique comprend:

14 a) un support sur lequel est posé «la préparation », constitué, le plus souvent, par la platine du dispositif d'observation ;

15 b) un système optique : microscope ou loupe binoculaire et l' éclairage correspondant ;

16 c) des micro-manipulateurs permettant de positionner des microélectrodes avec précision et de garder cette position; un micromanipulateur pneumatique de De Fombrune: trop sensibles aux variations de température, ces instruments ont été remplacés par des dispositifs à vis micrométriques, puis par des systèmes hydrauliques ou piézo-électriques; des microélectrodes : les microélectrodes permettent de relier le milieu cellulaire, où les charges électriques sont portées par des ions, à des conducteurs métalliques, où les charges sont transportées par des électrons. Il s'agit de tubes de verre étirés jusqu à un diamètre de pointe inférieur à $1 \mathrm{pm}$, d'où le nom de micropipettes, puis remplis d'une solution de KCl où plonge un fil d'argent chloruré. D'abord ébauchées à la main dans une flamme puis finies à l'aide d'une micro-forge, elles sont maintenant réalisées grâce à des machines automatiques.

Les appareils de mesure et d'enregistrement 
17 Les signaux cellulaires sont étudiés à l'aide d'un ensemble d'appareils de mesure électroniques.

a) Des amplificateurs

Produits par des cellules dont la résistance électrique propre est très élevée et recueillis par des électrodes elles aussi de résistance élevée, les signaux électriques cellulaires imposent l'usage d'amplificateurs à très haute résistance d'entrée. Ils ont été très marqués par les progrès de l'électronique et ont été l'objet de nombreuses réalisations locales. Toutefois, aucun amplificateur à tube électronique utilisé avant 1960 n'a été conservé au laboratoire.

b) L'oscilloscope

21 L'oscilloscope a été longtemps l'instrument clef de l'électrophysiologie car il permet de visualiser les réponses cellulaires en fonction du temps. Il met en jeu de nombreuses propriétés physiques : le déplacement des électrons dans le vide, la déviation de leur trajectoire par les champs électriques et la génération de lumière par fluorescence. Dans un tube vide d'air (tube cathodique), un faisceau d'électrons est focalisé sur un écran fluorescent où il forme un spot lumineux. Le signal mesuré (par exemple, le potentiel membranaire) est appliqué après une amplification appropriée à deux plaques générant un champ électrique vertical. Ce champ dévie le faisceau d'électrons et par suite le spot se déplace suivant une ligne verticale au gré de la variation du signal. Pour en faciliter l'observation, une tension croissant linéairement est appliquée à des plaques de déviation horizontale dont le champ électrique déplace le spot horizontalement. Lorsqu il atteint le bord droit de l'écran, cette tension s'annule ramenant instantanément le spot au bord gauche où le processus reprend.

Les oscilloscopes du laboratoire vers 1960 étaient de fabrication anglaise (Cossor) ou américaine (Dumont) ; on trouvait aussi des appareils, assez limités à un seul canal, de fabrication française (C.R.C.). Puis sont apparus des appareils américains à deux canaux comme le Tektronix 502 qui comportait des tubes d'entrée thermostatés pour améliorer la stabilité ou le Tektronix 565 équipé $d$ 'amplificateurs interchangeables (tiroirs) le rendant très flexible.

Plus tard sont apparus des oscilloscopes à écrans rectangulaires dont la rémanence était entretenue donnant un effet de mémoire un peu à la manière des "ardoises magiques »: le Tektronix 564 acquis vers 1967 puis le Tektronix 5100 apparu vers 1972-1973, et dont certains sont encore en usage. Avec le développement de l'électronique, des oscilloscopes numériques sont apparus : ils échantillonnaient les signaux à une cadence variable, convertissant chaque point en un nombre stocké dans une mémoire électronique. Nous avons utilisé, à partir de 1976, des Nicolet. Ces appareils avaient toujours un écran cathodique mais la mémoire électronique permettait d'examiner à loisir les signaux mémorisés, tout en rendant possible l'agrandissement et la mesure à l'écran; une interface avec laquelle il était possible de transférer les données vers un calculateur était disponible. Actuellement, on trouve des oscilloscopes entièrement numériques (Tektronix série TDS, par exemple).

c) Un système d'enregistrement

25 Jusquà l'apparition des oscilloscopes numériques, mesurer les données sur l'écran n'était pas aisé ; en outre, il fallait archiver. On a utilisé des appareils photographiques 
très simples qui prenaient une photographie de l'écran à chaque balayage. Le film était avancé d'une vue (à la main, au début) soit après chaque balayage, soit après un nombre variable de balayages. Un cône faisant office de support permettait éventuellement de travailler en pleine lumière. Les films étaient analysés, après développement, à l'aide d'agrandisseurs.

Pour examiner des événements lents à très basse fréquence, les enregistreurs oscillographiques sur papier étaient préférés. A côté des enregistreurs galvanométriques dont la plume décrivait une trace courbe, il y avait des enregistreurs rectilinéaires très lents en raison de l'inertie de leur équipage mobile. Des appareils à réponse assez rapide $(-1000 \mathrm{~Hz})$ ont cependant été réalisés avec un miroir, monté sur un galvanomètre, sur lequel se réfléchissait un faisceau U.V. qui impressionnait un rouleau de papier sensible. La trace laissée sur le papier était toutefois très pâle et relativement fugace.

On a donc utilisé assez longtemps des caméras, dérivées de l'industrie cinématographique, placées devant l'écran d'un oscilloscope. Ces appareils fonctionnaient soit en mode vue par vue, soit en défilement: le film avançait d'une manière continue formant l'axe des temps, le balayage horizontal de l'oscilloscope n'étant plus utilisé.

Ces appareils ont été concurrencés par la mise sur le marché vers 1968 de traceurs à équipage mobile asservi et à encre sous pression écrivant avec un très bon contraste sur du papier glacé (Brush). Leur assez bonne réponse (100 Hz sur $20 \mathrm{~mm}, 7 \mathrm{~Hz}$ sur 100 $\mathrm{mm}$ ) leur a valu un grand succès malgré leur coût élevé tant à l'achat qu à l'usage. Parallèlement, pour des signaux à fréquences plus élevées on a utilisé des magnétophones à bandes. Cependant, pour enregistrer aussi les fréquences basses et les niveaux continus, il a fallu attendre l'apparition de variantes à modulation de fréquence ${ }^{1}$.

L'avènement des magnétoscopes constitua un net progrès: l'utilisation de têtes magnétiques rotatives inscrivant des pistes obliques permettait de passer les fréquences élevées (-1,5 Mhz) de la télévision. Il faudra cependant attendre 1982-1983 pour que deux firmes japonaises (Sony et Nakamishi) développent des convertisseurs dits «P.C.M.» (pour Pulse Coded Modulation) capables de convertir des signaux analogiques en pseudo-images TV pour disposer d'un système d'enregistrement performant et, de plus, numérique: après une conversion analogique-numérique à raison de 44000 mesures par seconde, les bits composants chaque nombre, formant des points noirs (0) ou blancs (1), étaient rangés en lignes puis en images, la procédure inverse se produisant à la lecture. Ce principe se retrouve aujourd'hui dans les enregistreurs numériques à cartouche (Digital Audio-Tape) et les magnétoscopes dits «Hi-Fi » pour le son.

d) Un générateur d'impulsions ou stimulateur

La méthode d'étude habituelle des cellules nerveuses ou musculaires consiste à perturber l'état dit de repos par une stimulation électrique appropriée et d'enregistrer le retour à l'état initial. De nombreux stimulateurs ont été conçus dans les laboratoires en raison des caractéristiques particulières requises. Les premiers modèles ont utilisé des tubes électroniques (comme celui conçu par M. Pokrovski au Collège de France, ou par Grass Instruments aux Etats-Unis). Puis sont apparus des modèles à transistors mais 
toujours analogiques, achetés encore vers 1980, puis des modèles numériques à circuits intégrés vers 1970 (par exemple, Digitimer 4030).

Conclusion

Dans cette énumération manque un constituant essentiel de tout poste électrophysiologique actuel: l'ordinateur personnel apparu en 1981-1982 (Apple II, Victor Sirius). Non seulement il remplace aujourd'hui l'oscilloscope, le stimulateur, l'enregistreur, mais il peut piloter les amplificateurs qui restent l'intermédiaire obligatoire en raison des problèmes d'impédance évoqués plus haut. Grâce à des logiciels élaborés, il permet en outre des analyses et une réduction des enregistrements. Enfin, en plus des signaux de potentiel ou de courant, il peut enregistrer des images de fluorescence reproduisant l'activité des cellules étudiées.

En raison de cette évolution, et de la spécialisation, il devient de plus en plus rare de rencontrer des électrophysiologistes connaissant les principes physiques qui sont à la base des appareils décrits ci-avant alors que l'électrophysiologie se rattache, bien sûr, aux neurosciences mais aussi à la biophysique. Cet état de fait résulte ainsi de l'irrésistible développement de l'électronique et de l'informatique, mais sans doute aussi de la raréfaction des ingénieurs spécialistes en électronique dans les laboratoires de biologie, conjuguée au développement de la recherche qui fait que la construction d'appareils à l'échelle industrielle devient rentable. Il s'agit d'un appauvrissement certain car les interactions entre les différentes branches des sciences sont essentielles pour progresser.

Doit-on conserver ces appareils? Plusieurs considérations poussent à répondre affirmativement. Tout d'abord, ils témoignent de l'histoire de l'avancement de la technologie en liaison avec la connaissance fondamentale. En effet, il est clair que beaucoup d'avancées enregistrées dans les neurosciences, comme les modulations à long terme de la transmission synaptique ou la mise en évidence des canaux ioniques membranaires, sont étroitement liées aux améliorations de l'instrumentation. Ensuite, ils constituent aussi un lien entre les différentes branches de la science puisque le même appareil peut être utilisé dans des domaines assez différents. Enfin, ils témoignent de la génération des concepts et de l'imbrication des connaissances. Ils constituent donc des documents pédagogiques remarquables.

La sauvegarde des petits et grands instruments en physique nucléaire et en physique des particules (C)

Pas plus que l'homme préhistorique taillant ses silex ou allumant son feu, le chercheur, l'ingénieur ou le technicien n' ont conscience de la valeur historique des témoignages de leur activité. Entièrement tournés vers la réalisation de leurs expériences et la publication de leurs résultats, ils n'ont généralement pas le réflexe de sauvegarder leurs documents, ni leurs instruments, lorsqu'ils deviennent obsolètes. L'attitude la plus courante consiste à stocker ces appareils dans un coin du laboratoire, en attendant de s'en débarrasser, après en avoir éventuellement récupéré les pièces encore utilisables. Parfois, cependant, certains de ces scientifiques éprouvent le désir de sauvegarder l'objet qui a été leur compagnon de recherche pendant de nombreuses années. Il s'agit alors d'initiatives d'individus ou de petites équipes, parfois encouragées par la direction du laboratoire concerné, mais qui se heurtent toujours au délicat problème des moyens en crédits, personnel et locaux. Il n'existe en effet aucune 
organisation officielle pour ces opérations de sauvegarde de notre patrimoine scientifique. C'est à l'examen de ce problème général, qui concerne archives et instruments, que s'est attaqué le groupe de réflexion pour la sauvegarde du patrimoine scientifique de l'IN2P3, dans le cadre des travaux menés par le Comité pour l'histoire du CNRS, et coordonné par Catherine Nicault et Henri Ostrowiecki. Cet exposé s'appuie sur l'étude menée par ce groupe de réflexion [voir bibliographie 1], ainsi que sur quinze années d'expérience personnelle en matière de sauvegarde d'instruments et de communication scientifique.

Petits et grands instruments

Une partie importante du patrimoine scientifique de la physique nucléaire et de la physique des particules est constituée de très grands instruments, accélérateurs ou détecteurs. Les plus volumineux d'entre eux ${ }^{2}$ appartiennent à des centres internationaux, comme le CERN, organisation européenne pour la recherche nucléaire, et leur sauvegarde doit s'inscrire dans un cadre international. Cependant, des machines locales, régionales ou nationales, de taille tout à fait respectable fonctionnent ou ont fonctionné en France. A Grenoble, par exemple, l'ensemble régional SARA, constitué de deux cyclotrons, a été arrêté récemment, et le problème de sa sauvegarde ou de son démantèlement est posé. Mais il existe aussi nombre d'instruments de dimensions plus réduites, dont la sauvegarde présente également un grand intérêt, mais s'avère beaucoup moins compliquée. Dans cette catégorie, on peut citer certains accélérateurs électrostatiques, des maquettes ou des éléments d'accélérateurs, et tous les types de modules équipant les grands détecteurs (scintillateurs solides ou liquides, diodes au germanium et au silicium, compteurs Geiger ou Cerenkov, détecteurs multifils de type Charpak, etc.). Un exemple intéressant est celui des chambres d'ionisation, inventées par les Curie en 1897, et qui n'ont cessé d'évoluer jusqu aux modèles équipant actuellement le multi-détecteur INDRA.

Des exemples de sauvegarde

En ce qui concerne la physique nucléaire, il faut d'abord rendre hommage à l'action considérable menée depuis des années par l'Association Curie et Joliot-Curie, qui se traduit par l'existence du Musée et des Archives Curie, relevant conjointement de l'IN2P3 et de l'Institut Curie. Les documents et le matériel qui s'y trouvent rassemblés couvrent une durée de plus de soixante années, depuis les premiers travaux de Pierre et Marie Curie (1897) qui jetèrent les bases de cette discipline, jusqu'aux dernières actions d'Irène et Frédéric Joliot-Curie (1958) qui en assurèrent l'essor.

38 A Strasbourg, l'accélérateur électrostatique de type Cockroft-Walton, construit en 1942-1944 à l'initiative des occupants allemands dans le but de synthétiser des isotopes radioactifs, et ayant fonctionné pour la recherche de 1946 à 1959, a été conservé et est exposé sur une pelouse de l'IRES (Institut de recherches subatomiques) [2, 3].

Sur le site d'Orsay, l'accélérateur linéaire LINAC, qui, associé à un cyclotron, constituait l'ensemble ALICE (1966-1985), est exposé sur le campus depuis son classement comme monument historique en 1987. L'ensemble ALICE n'a pu être entièrement conservé, mais un film retrace son histoire en la replaçant dans le contexte socio-économique de l'époque [4]. Chaque année, lors de la journée du patrimoine, des visites guidées du LINAC sont organisées par l'Institut de physique nucléaire (IPN) d'Orsay, avec projection de ce film. 
40 En physique des particules, la chambre à bulles Gargamelle, dont l'exploitation conduisit, en 1973, à la découverte des "courants neutres ", étape essentielle pour la théorie de l'unification des forces [5], est exposée sur le campus du CERN.

41 A Orsay, une solution originale a été trouvée pour la sauvegarde de l'anneau de collision (ACO), qui fonctionna pour la recherche sur les particules élémentaires au Laboratoire de l'accélérateur linéaire (LAL) de 1964 à 1988, puis pour l'utilisation du rayonnement synchrotron. Un véritable musée, Sciences ACO, a été créé et cette expérience inédite est présentée ici par Pierre Dhez et Pierre Marin.

Vers une politique de sauvegarde

42 Compte tenu de l'évolution des programmes de recherche et des moyens techniques, nombre d'instruments, petits ou grands, doivent en permanence être réformés. Il semble donc indispensable de mettre sur pied une méthode permettant de sauvegarder ceux qui doivent (et qui peuvent) l'être. Dans cet objectif, un certain nombre de principes peuvent être dégagés.

43 En premier lieu, la sauvegarde d'un instrument donné doit s'accompagner de la constitution d'un dossier complet, scientifique et technique, rédigé dans des termes accessibles aux non-spécialistes, et regroupant documents imprimés, photographies, et éventuellement films, vidéos, cédéroms... De plus, chaque fois que possible, elle devra s'effectuer dans une perspective de mise en valeur immédiate tournée vers le grand public, et intégrée à la vie du laboratoire et de la région, plutôt que se limiter à un simple stockage en lieu sûr. En effet, malgré son indéniable intérêt pour l'histoire des sciences, qui la rend indispensable en l'absence d'autre solution, une telle opération de stockage risque de se révéler peu motivante. La sauvegarde du patrimoine scientifique apparaît ainsi comme une action pluridisciplinaire, dont l'initiative revient, bien entendu, aux scientifiques de la discipline et du laboratoire concernés, mais qui peut également faire appel à des spécialistes de l'histoire des sciences, de la pédagogie et de la communication scientifique. Toute action de sauvegarde doit donc s'effectuer dans un cadre institutionnel. Un tel cadre n'existe pas aujourd'hui, et sa création pourrait s'inscrire parmi les objectifs du Comité pour l'histoire du CNRS. Il paraît également nécessaire d'établir une coordination à 'échelle de chaque discipline pour établir les inévitables priorités. A la demande du groupe de réflexion sur le patrimoine scientifique de l'IN2P3, les laboratoires de cet Institut ont récemment mis en place des correspondants en patrimoine, ce qui constitue un premier pas très important vers cette politique de coordination.

Un arsenal de solutions

44 Face à la grande variété des appareils susceptibles d'être sauvegardés, il est évident que tout un arsenal de solutions doit être mis en oeuvre, chacune d'elles tenant compte des dimensions de l'appareil, de la situation du laboratoire qui en prend l'initiative, de son insertion dans le tissu régional, des partenariats qui seront mis en oeuvre et des moyens (crédits, locaux et personnels) qui pourront être dégagés.

45 On peut imaginer, par exemple, qu'un grand instrument, dont la construction aurait été cofinancée par une région, soit intégralement conservé et devienne, à l'instar de Sciences ACO, un lieu régional de rencontres scientifiques, une sorte de « Maison de la science ", appartenant désormais au patrimoine d'une région. Bien entendu, une telle sauvegarde relève de l'exploit, et des solutions plus modestes, impliquant la 
conservation de certaines parties de l'appareillage, complétée par la réalisation de documents filmés, devront souvent être mises en place pour conserver la mémoire de ces grands instruments. Un simple dossier photographique, bien organisé et convenablement légendé, dont les éléments seraient issus des photothèques de l'IN2P3, du CNRS et du laboratoire, constituerait déjà une sauvegarde précieuse. Il conviendrait $d$ 'ériger en règle la constitution d'un tel dossier pour tout instrument cessant de fonctionner.

Pour les petits appareils, l'éventail des solutions s'élargit. En effet, on peut alors concevoir, ce qui se pratique couramment dans les laboratoires, l'exposition de ces appareils dans des vitrines situées dans les halls, couloirs, ou, comme au LAL d'Orsay, dans une salle dédiée à ce type d'exposition. L'aménagement du hall d'entrée du bâtiment $\mathrm{K}$ au siège du CNRS à Paris, rue Michel-Ange, lorsque l'IN2P3 s'y est installé en 1993 [6], constitue un exemple original. En face de vitrines exposant du petit matériel (détecteurs), on peut y trouver un bas-relief évoquant de façon stylisée le cyclotron du Collège de France.

On peut également concevoir que des appareils légers, conservés en état de fonctionnement ou non, soient utilisés à des fins pédagogiques lors de la visite des laboratoires par des classes de lycéens, ou soient emportés par des conférenciers pour illustrer des conférences grand public ou dans l'enseignement secondaire ou supérieur. Ce type d'action peut se révéler très efficace pour éveiller des vocations scientifiques chez les jeunes.

Enfin, parmi les musées nationaux ou régionaux, certains peuvent accueillir des objets de dimensions relativement modestes au sein de leurs expositions permanentes ou temporaires.

Dans tous les laboratoires, des potentialités existent. Des chercheurs, ingénieurs et techniciens sont prêts à agir dans le domaine de la sauvegarde du patrimoine scientifique. Des directions de laboratoires sont prêtes à les soutenir. Au-delà des recommandations et des conseils qu'il serait nécessaire de diffuser largement et fréquemment, le plus urgent est de donner à ces actions une reconnaissance officielle au niveau de l'IN2P3 et du CNRS, et de prévoir un minimum de moyens en personnel et en crédits pour le lancement des opérations ayant quelque envergure. Notons qu'il s'agira ici, en général, de moyens incitatifs, donc relativement faibles, de telles opérations devant requérir obligatoirement un financement au-delà des organismes de recherche.

Historique et préservation du développement instrumental à l'Institut d'optique (D)Introduction

50 Pourquoi cette idée d'un regroupement d'instruments illustrant l'histoire de l'Institut d'optique (IOTA) ? La nécessité d'un tel «mini-musée » s' est progressivement imposée dans l'esprit des membres du laboratoire, et le projet commençait précisément à faire l'objet de discussions internes lorsque l'initiative d'une opération de mise en valeur et de préservation du patrimoine instrumental est venue de notre établissement de tutelle, le CNRS. Assurément, chaque discipline, chaque branche, des grandes disciplines scientifiques, a son histoire et son approche : nous sommes donc heureux de profiter de cette occasion pour témoigner, à travers le cas particulier de notre institut, de la situation de l'optique, dont l'IOTA est un des foyers français depuis plus de quatre- 
vingts ans. Ses instruments font partie de son histoire et même, dans une certaine mesure, résultent directement de sa mission, comme nous le rappellerons ci-après : nombreux sont les prototypes, les têtes de séries, les dispositifs originaux qui ont vu le jour dans nos murs; à travers eux, on peut distinguer les grandes périodes du développement de l'optique depuis 1920.

Aperçu historique

51 C' est en effet dès 1915, en pleine Grande Guerre, qu'Armand de Gramont, industriel d'instruments de précision alors sous les drapeaux, se rend compte du besoin en instruments d'optique. Avec le professeur Henri Chrétien, célèbre pour son cours de calcul des combinaisons optiques, il établit le projet d'un institut formant des ingénieurs capables de calculer les éléments de l'optique instrumentale. Les circonstances sont favorables au projet: le portefeuille du ministre de l'Instruction publique et des Beaux-Arts s'étendait pendant cette période de guerre aux « inventions intéressant la Défense nationale ", comme le montre l'en-tête de l'invitation adressée par le ministre Paul Painlevé à ses collègues de la Guerre, de la Marine et du Commerce pour « jeter les bases d'un Institut d'optique » en présence d'A. de Gramont, qui sera ensuite appelé à présider le conseil du nouvel établissement. S'il faut ensuite attendre plus de trois ans pour que le rapport de la commission parlementaire désignée pour proposer des statuts soit suivi d'effet, c'est bien en lien direct avec la recherche universitaire qu' en 1920 l'Institut d'optique théorique et appliquée (IOTA) se voit confier au service de l'optique une triple mission :

- contribuer à l'avancement des connaissances,

- former des ingénieurs et des ouvriers qualifiés,

- et constituer un centre d'études et d'essais.

Le lien entre recherche et besoins sociaux, économiques et politiques, est de la sorte inscrit dans nos statuts. Le premier directeur général est Charles Fabry, professeur en Sorbonne et inventeur, avec Alfred Perot, d'une configuration d'interféromètre dont l'importance devait s' avérer primordiale pour la conception des lasers. Depuis lors, une convention définit les relations entre l'IOTA et l'Université (depuis 1972, l'université Paris-Sud) et les relations ont été permanentes entre les laboratoires de recherche de I'IOTA et son environnement universitaire, dans lequel est apparu le CNRS. Les industriels de l'optique participent de façon déterminante au conseil d'administration de l'IOTA et les services de l'Armement, entre autres organismes, lui confient des études liées en particulier à l'instrumentation. D'où le développement d'un grand nombre d'instruments originaux dont nous citerons ci-après quelques exemples en distinguant trois périodes : avant la Seconde Guerre mondiale, entre cette dernière et l'invention du laser, et depuis l'invention du laser.

Trois périodes du développement d'instruments à l'Institut d'optiqueEntre les deux guerres

53 Dans ses premières années, l'IOTA se consacre largement à la conception de nouveaux instruments de mesure pour les quantités de base intervenant en optique: angles, indices de réfraction, rayons de courbures, grandeurs photométriques, spectroscopie... Les vérifications et mesures portent sur l'étude des « substances » (on ne dit pas encore matériaux), celle des formes, la détermination des aberrations, le contrôle des 
appareils. Destinées à être transférées à l'industrie, les activités des laboratoires relèvent de celles d'un centre technique mettant à la disposition des industriels de multiples moyens de contrôle et d'expertise. En même temps, elles se situent à la pointe des connaissances et sont publiées dans les revues de l'époque. Alors que certains appareils sont par leur principe pionniers en leur genre, d'autres sont si précis qu'ils restent en usage de nos jours avec l'agrément du Comité français d'accréditation.

Cette période peut être illustrée par deux appareils préservés par le temps: le vérificateur de sextants (1924) mis au point par Ch. Fabry et le colonel Charles Dévé et qui vérifiera tous les sextants utilisés par le service hydrographique de la Marine; les archives nous sont restées pour la période postérieure à 1947 : en 32 ans, jusqu'à la cessation de cette activité en 1979, 796 sextants passèrent au service de métrologie de l'IOTA avant de servir aux officiers de la Marine.

Un autre exemple est le banc servant à la mesure des rayons de courbure, élaboré par Albert Arnulf (1930) et fort utile pour les industriels devant réaliser avec précision les lentilles qui sortent des feuilles de calculs fastidieux des spécialistes des combinaisons optiques. Ces derniers étaient faits à la main avec les tables de logarithmes à sept décimales, et il n'était pas question, comme on le fait aujourd hui, d'adapter le calcul à la mesure mais bien de réaliser rigoureusement le résultat du calcul, quitte à multiplier les retouches et donc les mesures!

Après 1945 et avant le laser

L'optique physique se développe et ses propriétés sont de plus en plus utilisées dans la conception des instruments. Des chercheurs du CNRS se joignent aux membres de l'enseignement supérieur, renouvellent et diversifient les recherches, tout en leur laissant une vocation essentielle d'instrumentation: citons l'invention de la microscopie interférentielle, destinée à l'observation des objets de phase, qui doit beaucoup à Georges Nomarski et à Maurice Françon, le calculateur analogique électromécanique d'André Maréchal qui traçait les figures de diffraction d'un instrument entaché d'aberration, et encore le rôle pionnier de Florin Abéles dans la conception des empilements de couches minces, destinés aux traitements réfléchissants ou anti-reflet.

De cette période, nous avons retenu pour notre mini-musée l'endoscope universel de Jacques Vulmière (1951), adapté à l'observation de l'intérieur d'objets manufacturés, aussi bien qu'à certains examens médicaux, et l'interféromètre à référence ponctuelle (1964) de G. Nomarski.

La révolution du laser

Lorsqu'en 1966, les laboratoires de l'IOTA font partie de la première vague de reconnaissance de laboratoires associés par le CNRS, le laser y a fait son entrée. En quarante ans, il bouleversera l'optique au point que des néologismes comme " optoélectronique ", " photonique » et " optronique », au demeurant assez mal définis, témoignent de la difficulté que l'on éprouve parfois à reconnaître dans l'optique actuelle le substrat d'avant 1960, qui pourtant y subsiste en totalité.

Cette période se poursuit de nos jours, et il ne se conçoit plus guère d'instrument qui ne soit d'une façon ou d'une autre associé au laser. Mais certains instruments ayant joué un rôle historique seront retenus pour notre mini-musée. Le polariseur à piles de glaces (1978) a fait partie de l'expérience par laquelle Alain Aspect a tranché entre 
l'interprétation de Copenhague de la mécanique quantique et les théories causales à variables cachées locales. L'interféromètre pour photons uniques (1985) de Philippe Grangier a établi, de façon beaucoup plus concluante que les expériences précédentes, la formation de franges d'interférences par un photon unique traversant un interféromètre. Dans cette expérience, une utilisation convenable du laser permet d'assurer l'unicité de ce photon. Le laser femtoseconde (1986) conçu par François Salin et Gérard Roger, capable de fournir des impulsions de durée 50 femtosecondes (Ifs = $10^{-15}$ s), et sur lequel a été démontré le comportement «solitonique » des impulsions produites, est le précurseur d'un modèle qui sera commercialisé grâce aux travaux de Patrick Georges.

Le regroupement des instruments

60 A l'IOTA comme partout, la place fait défaut et dans les placards les vieux instruments subissent les outrages du temps. La tentation de se débarrasser des instruments devenus obsolètes ou hors d'usage revient périodiquement, lorsqu'ils ne sont pas démontés pour que leurs pièces détachées soient intégrées aux expériences nouvelles (voire transformées en aquarium, destin imprévu que connaît une ancienne cloche à vide!). Certains pourtant sont encore intacts et peuvent être utilisés dans des démonstrations pour faire revivre un aspect de l'histoire des sciences et des techniques. Voilà pourquoi nous avons voulu procéder à un regroupement de tels appareils : provisoirement regroupés dans notre salle de réunions "Charles Fabry ", notre "mini-musée » sera ouvert aux visiteurs intéressés. Il reste à compléter le financement pour le présenter de façon didactique et agréable, et à prévoir son implantation dans notre futur bâtiment, prévu pour 2004, de sorte à inciter le plus grand nombre à s'y instruire à la lumière du passé.

Le patrimoine scientifique : un exemple de valorisation et de collaboration entre un organisme de recherche et un CCSTI (E)

61 À l'aube du $3^{\mathrm{e}}$ millénaire, le CNRS situé à Orléans sur le campus de la Source a décidé d'organiser une exposition d'objets scientifiques avec la participation du centre de culture scientifique, technique et industrielle (CCSTI) de la région Centre, CentreSciences. Cette exposition est installée au Muséum des sciences naturelles d'Orléans depuis le 2 mai 2001 et a été inaugurée en présence d'Hubert Curien, président de l'Académie des sciences. L'exposition, qui devait initialement se terminer le 23 mai, a connu un vif succès, et elle a été prolongée jusqu à la fin juin 2001.

L'idée de cette exposition tire son origine d'un projet de manifestation qui devait être organisé en l'an 2000 au pied de la tour Eiffel par le CNRS : «Question de savoir ou oser le savoir » et qui ne s'est pas concrétisé. Il visait à réunir un maximum d'objets ayant appartenu aux laboratoires du CNRS. Comme cette première initiative n'a rencontré aucun écho auprès des directeurs quant à l'idée de voir leurs instruments partir à Paris, j'ai alors souhaité réaliser cette exposition à Orléans et, là, les objets sont timidement sortis des placards et des caves où ils dormaient. Puis $\mathrm{j}$ ai recherché un partenaire pour réaliser ce projet et je l'ai rencontré avec Centre-Sciences.

63 Pourquoi avoir contacté uniquement des laboratoires d'Orléans alors que la délégation Centre-Auvergne-Limousin comprend des laboratoires situés également à Tours, Limoges et Clermont-Ferrand? Tout simplement à cause d'un problème de logistique. En effet, ce travail a pu être réalisé grâce à un réseau de correspondants de 
communication au sein des laboratoires d'Orléans (constitués par des chercheurs, des ingénieurs, des techniciens et des gestionnaires de laboratoire). Ils sont ainsi partis en quête d'objets dans chacune de leurs unités de recherche.

Ces objets ont été photographiés et j'ai fait établir une fiche d'identité pour chacun d'entre eux contenant les informations indispensables suivantes : le nom, la taille et le poids, l'histoire, le créateur, l'usage (à quoi sert-il ?) et l'évolution (a-t-il son équivalent moderne ?). Au départ, avec Centre-Sciences, nous avons sélectionné cinquante objets en veillant à n'oublier aucun laboratoire du Campus. Pour le choix du lieu, ne possédant aucune salle adéquate sur le Campus du CNRS, nous avons cherché une localisation en ville. Notre premier choix a été tout d'abord d'aller nous installer dans le nouveau Centre de conférences qui s'est ouvert en mars 2001. Mais, pour des raisons budgétaires, nous avons sollicité le conservateur du Muséum qui, intéressé par notre initiative, nous a proposé une salle. Le Muséum des sciences naturelles est un très beau musée dans lequel on trouve :

- une collection importante de cristaux qui provient du Bureau de recherches géologiques et minières (BRGM),

- des insectes, vivariums et aquariums,

- des mammifères et des oiseaux,

- une serre tropicale et une serre méditerranéenne.

Il y a également deux salles qui permettent de réaliser des expositions. L'une d'elle, primitivement destinée à être un planétarium, est restée inoccupée, et c'est cette dernière qui nous a été proposée.

Nous avons donc essayé de tirer parti de la configuration circulaire de cette salle pour organiser l'exposition.

L'exposition a été organisée de façon à permettre à des visiteurs non familiarisés de découvrir des objets en tant que tels, de sorte qu'ils puissent se faire une idée de l'environnement de travail des scientifiques quelques années auparavant. En contrepoint de ces objets, $j$ ai disposé des panneaux photographiques où figurent des hommes et des femmes travaillant aujourd'hui dans les laboratoires avec des appareillages modernes.

Les objets sont disposés dans les vitrines de manière que leurs qualités esthétiques et leur caractère insolite soient aussi évidents que possible pour le visiteur. Le but était moins de lui faire faire un parcours didactique que d'aiguiser sa curiosité.

Un autre objectif recherché était de montrer le savoir-faire de ceux qui ont conçu et construit ces appareils, de faire ressortir le fait que l'activité scientifique demande du soin, qu'elle cherche à répondre à des défis et que ces appareils en sont un peu la matérialisation.

Cette exposition répond donc premièrement au souci de sauvegarder un patrimoine $\mathrm{d}$ 'appareils scientifiques qui sont les témoins de la science $d u \mathrm{XX}^{\mathrm{e}}$ siècle. Elle essaie ensuite d'utiliser ce potentiel à des fins éducatives, parce qu'il permet de se représenter, plus facilement qu'avec les moyens sophistiqués d'aujourd'hui, ce qu' est une démarche scientifique. C' est un moyen aussi de favoriser l'accès d'un large public à une représentation visuelle de ce qu est la recherche, plutôt qu'à travers les manuels 
scolaires. Ce public peut ainsi mieux appréhender des notions dont il a besoin en tant que public-citoyen devant participer à des décisions qui engagent son propre avenir.

Tous les objets (au nombre de 70) que nous avons rassemblés proviennent des laboratoires du CNRS situés sur le campus du CNRS et de l'université d'Orléans. Deux de ces objets proviennent de la station de radioastronomie de Nançay. La particularité d'Orléans est que l'éventail des disciplines scientifiques y est bien représenté puisqu'il va des sciences humaines aux mathématiques, en passant par la biologie, la physique, la chimie ainsi que les sciences de la Terre et de l'espace. Un certain nombre de ces laboratoires a été créé à la fin des années 1960, tandis que d'autres ont été délocalisés à partir d'autres régions. Ces laboratoires ont apporté avec eux des instruments qui ne sont plus utilisés aujourd' hui, mais qui témoignent de l'histoire de ces disciplines et de ce campus.

Ce retour sur le passé est en fait très lié au présent et à l'avenir, puisque nous avons voulu faire figurer, en contrepoint des vitrines d'exposition, des documents photographiques représentant des scientifiques d'aujourd'hui, mais aussi des photos montrant des résultats d'expériences ou des observations, en faisant ressortir la dimension esthétique des résultats de ce travail quotidien.

73 Nous avons regroupé ces objets en essayant de les présenter dans un contexte dynamique, et pour cela nous les avons répartis en quatre types d'activité qui consistent à :

- expérimenter,

- observer,

- mesurer,

- mémoriser, classer ou ordonner.

Nous avons tenté de faire sentir au public que l'on cherche à se réapproprier des forces qui n'apparaissent pas de manière évidente dans notre monde quotidien, ou par exemple à recréer des environnements qui n'existent que dans des univers lointains. Ainsi, le monde scientifique permet de faire rêver, d'inciter les gens à sortir, à s'extraire de leur monde quotidien pour découvrir d'autres apparences de la réalité, sans quitter le monde rationnel.

Lors de l'inauguration de cette exposition, Hubert Curien a exprimé plusieurs idées au sujet de ces appareils. D'abord, le souvenir de ses collaborateurs et des maîtres qui l'ont initié à la recherche.

Mais aussi, la réflexion que la science, qui était déjà bien avancée il y a un demi-siècle, a parcouru un chemin encore plus considérable depuis.

77 Qu'il me soit permis pour conclure d'estimer que la vision rétrospective stimule la réflexion sur les rapports entre l'évolution des connaissances et celle de la société, facilitant ainsi le questionnement sur leur devenir. Plus précisément, la description extensive de la science d'aujourd'hui ne peut suffire à elle seule à faire comprendre toute la dimension humaine de la recherche, et le regard sur le passé est nécessaire pour acquérir le recul et la profondeur de vue indispensables.

L'anneau de collisions d'Orsay : un exercice de muséographie (F)

Dès la fin de la Seconde Guerre mondiale, la physique nucléaire et la physique des particules furent l'un des domaines de recherches ayant fait l'objet d'un fort 
développement et d'une coordination à l'échelle nationale et internationale. A partir de l'effort initial de Frédéric Joliot-Curie, et en s'inspirant du concept de Big Science mis au point aux Etats-Unis, la France organisa son propre programme. Ainsi, à des fins civiles mais avec des arrière-pensées militaires, plusieurs accélérateurs ont été construits, en particulier à Orsay et sur le plateau proche de Saclay. Cinquante ans plus tard, nombre de ces machines sont arrêtées. Et, comme cela a été le cas dans certains secteurs industriels, se pose aujourd'hui la question de sauvegarder quelques machines nationales, témoins de cette époque. Pour les recherches fondamentales, les études sur les particules se poursuivent généralement avec des accélérateurs organisés dans des cadres internationaux, par exemple au CERN pour l'Europe.

79 Par son rôle scientifique et son environnement, l'anneau de collisions d'orsay (ACO) est un cas particulièrement intéressant. Il est situé au coeur d'une des plus grandes universités scientifiques françaises. Il est un exemple de reconversion réussie puisqu i il a aussi été une source de lumière synchrotron et a abouti à la création du LURE (Laboratoire d'utilisation $\mathrm{du}$ rayonnement électromagnétique), un laboratoire pluridisciplinaire dans lequel se pratiquent la physique, la chimie et la biologie. Le site du LURE sera prochainement modifié, lors de la construction de la nouvelle source de rayonnement synchrotron SOLEIL à Saclay, d'où la relative urgence de décider de sa sauvegarde afin d'éviter un démantèlement irréversible.

L'anneau de collisions d'Orsay (ACO) a été initialement conçu pour être un anneau de stockage d'électrons et de positrons afin d'étudier les produits de la collision entre ce couple particule-antiparticule. D'une dizaine de mètres de diamètre, il fut construit entre 1962 et 1965 dans une salle d'environ $200 \mathrm{~m}^{2}$ par le Laboratoire de l'accélérateur linéaire (LAL) comme complément de l'accélérateur linéaire. Il est encore d'une dimension humaine, analogue à celle d'un petit observatoire astronomique, contrairement aux collisionneurs modernes tels que ceux du CERN qui atteignent la taille des boulevards périphériques de Paris. Mais ces gigantesques machines assurent les mêmes fonctions que leur ancêtre ACO : source de particules, accélération, stockage, collisions observées par un ensemble de détecteurs. $S$ 'il fut le premier collisionneur à être construit en Europe pour la physique des particules, ACO fut aussi le premier anneau de stockage à être employé dès 1973 comme source de rayonnement synchrotron. Partagé tout d'abord avec les physiciens des particules, il fut ensuite entièrement dédié à la production de rayonnement jusqu'à son arrêt en 1988, après la construction de SuperACO. Le rayonnement synchrotron est émis par des particules chargées circulant sur une trajectoire courbe ; il fut initialement considéré comme une perte d'énergie pour les collisionneurs. En fait, depuis le domaine infrarouge jusqu'aux rayons $\mathrm{X}$, ce nouveau type de sources est maintenant reconnu comme la plus brillante. Depuis cette époque, beaucoup de pays se sont construit leurs propres machines. Elles permettent de sonder les atomes et les matériaux à différentes énergies; par exemple, les biologistes l'utilisent pour étudier la structure des protéines.

81 Alors que l'anneau ACO a été gardé dans son état original depuis son arrêt, la salle dans laquelle il fut construit a été régulièrement aménagée depuis 1994 pour faciliter et développer des visites de publics divers. Sciences ACO, une association de type loi de 1901 créée en 1994, veille à ces développements. Certains de ses membres, des bénévoles, assurent les visites du site pour les groupes scolaires, et aussi pour un public 
plus large lors de manifestations comme la fête de la Science, ou à la demande d'organismes divers comme le Syndicat du tourisme de la Vallée de Chevreuse. ACO accueille un millier de visiteurs chaque année.

La photographie montre l'appareil dans son état actuel. Au centre de la section droite principale, un simulateur de détecteur de traces de collision a été installé pour présenter la fonction initiale de collisionneur. Quelques panneaux d'information, ainsi qu'une "fresque sonore », permettent aux visiteurs de situer la machine sur l'ensemble $\mathrm{du}$ site du LAL et offrent quelques repères historiques des vingt-cinq années de développement. Par ailleurs, des détecteurs de particules, anciens ou en fonctionnement sur des machines actuelles ainsi que des prototypes pour de futures expériences, illustrent l'évolution des dispositifs, en taille et en technologie. Deux ordinateurs équipés de logiciels interactifs et un ensemble vidéo sont aussi en permanence dans la salle. Ces équipements ont été acquis avec l'aide de laboratoires proches et intéressés par ce site (LURE, LAL, IN2P3, DAPNIA, DRECAM...). Deux études muséographiques, l'une en 1994 et l'autre en 2000, ont été financées par le ministère de la Recherche pour identifier les aménagements possibles et évaluer les coûts. Si nécessaire, une extension sur des salles proches peut être envisagée et permettrait d'optimiser l'espace d'exposition, d'installer des services éventuels, ou même d'envisager d'y regrouper des activités culturelles actuellement disséminées sur le campus d'Orsay.

L'expérience pédagogique acquise par Sciences ACO depuis quelques années a permis d'identifier les contraintes et les possibilités de ce nouveau type de lieu, à la fois témoin du passé et en contact avec les développements scientifiques actuels de la physique des particules et l'utilisation du rayonnement synchrotron. Dans notre société en évolution constante, ACO apparaît comme un exemple permettant d'illustrer les liens entre les recherches fondamentales et appliquées. Il peut aussi aider à faire comprendre les différents aspects du nucléaire. En effet, ce dernier, comme nous l'avons constaté, est particulièrement mal perçu par la quasi-totalité des visiteurs, qu'ils soient scolaires, étudiants ou visiteurs extérieurs à la recherche.

ACO est situé dans un campus universitaire destiné à être réaménagé dans les années à venir. Dans ce plan, un projet de parcours scientifique est à l'étude ; ACO en serait l'un des points d'intérêt. De plus, cette université possède un Groupe d'histoire des sciences, qui forme des étudiants à l'histoire des Sciences, et un Centre de vulgarisation des connaissances (CVC). Elle héberge aussi un Centre de culture scientifique, technique et industrielle (CCSTI sciences ressources) qui pourra servir de point d'appui. Cet environnement riche est une occasion à saisir pour tenter de créer bien plus qu un musée témoin des quarante dernières années de la recherche.

Ce lieu dont la vie scientifique et les reconversions successives sont exemplaires permet de montrer, d'illustrer et d'expliquer les liens, ainsi que l'histoire de l'évolution des différents aspects et besoins sociaux de la recherche. Des spécialistes des questions du patrimoine scientifique semblent s'orienter vers ce type de formule plus liée à la société. On parle alors d'une approche « sciences, technologies et sociétés » (STS) 3 .

Un ensemble de partenaires est évidemment nécessaire pour décider de la forme muséographique ainsi que du type d'organisation et des tutelles devant ensuite assurer gestion et financement. Il nous apparaît indispensable que les acteurs suivants 
participent: CNRS, ministère de la Culture, Région Ile-de-France, Direction régionale des affaires culturelles (DRAC), Département, université Paris-Sud et, bien entendu, la future source synchrotron SOLEIL.

Enfin, le démantèlement des installations prévu dans les prochaines années exige une prise de décision urgente quant à une sauvegarde minimale du site. C' est la raison pour laquelle une demande de classement d'ACO par les Monuments historiques est à l'étude pour assurer sa pérennité.

Intérêt des maquettes et prototypes en physique des particules (G)Introduction

Dans le cadre des conférences-débats du Comité pour l'histoire du CNRS, traitant de la sauvegarde du patrimoine instrumental, je voudrais montrer l'intérêt pédagogique que revêt, dans le domaine de la physique subatomique, la réalisation de maquettes et de prototypes pour illustrer notre discipline ; je ferai ensuite le lien avec la sauvegarde des détecteurs, et les difficultés que l'on peut rencontrer pour trouver des locaux et des moyens, tant humains que financiers, même lorsqu' il y a un accord sur la conservation de ce patrimoine.

La première partie vise plutôt à exposer la méthodologie de la collecte patrimoniale. La seconde est consacrée à des exemples pour mieux souligner les difficultés rencontrées.

Maquettes et prototypes : dans quel but?

La physique subatomique, qui regroupe les disciplines concernant l'étude des noyaux et celle des constituants élémentaires de la matière (particules et astroparticules), s'intéresse à l'infiniment petit et à ses liens avec l'infiniment grand (étoiles, objets galactiques).

91 Pour "voir l'invisible», des détecteurs capables de reconstituer les trajectoires des particules ou de mesurer leur énergie sont utilisés. Mais plus on veut sonder la matière profondément, pour reconnaître de petits objets, plus l'énergie à fournir est grande (ce qui implique d'utiliser des grands complexes d'accélérateurs) et plus les détecteurs doivent être sensibles et nombreux (donc, de grands instruments de mesure). La physique subatomique est donc une physique qui utilise des grands instruments, ce qui va motiver la construction de prototypes, ainsi que la réalisation de maquettes à but pédagogique, pour remplacer les détecteurs que l'on ne peut montrer.

Le problème de la réalisation des détecteurs

92 En physique subatomique, la plupart des expériences sont le fruit de grandes collaborations internationales.

93 A l'heure actuelle, aucun laboratoire, et ce quel que soit son pays d'origine, n'est capable de construire seul un détecteur de physique subatomique. Dès lors, le détecteur final a de multiples concepteurs et propriétaires, et souvent dans différents pays. Dans l'idée de sauvegarder le patrimoine instrumental français, il parait important de définir à l'avance à qui appartiendra le détecteur, ou une partie du détecteur, lorsque l'expérience sera terminée.

94 Dans le même état d'esprit, ces grands détecteurs sont formés de multiples sousdétecteurs : détecteur de vertex, détecteur de traces, calorimètre électromagnétique et calorimètre hadronique, bobine magnétique, détecteur à muons, blindage... Toute cette énumération de sous-détecteurs ne représente en fait qu un seul gros détecteur de type classique utilisé en physique des particules, par exemple pour les recherches effectuées 
auprès du collisionneur LEP (Large Electron-Positron Collider) du CERN. Ces différents sous-détecteurs sont en général réalisés en différents endroits et, souvent, chacun d'eux est lui-même segmenté, et il est finalement difficile pour les différents laboratoires $\mathrm{d}$ 'avoir une vue d'ensemble du détecteur complet à présenter, puisque ce dernier est souvent assemblé sur le site même de l'expérience.

Enfin, ces sites d'expériences en physique subatomique sont en majorité éloignés des laboratoires, les grands complexes d'accélérateurs qui accueillent ces grands détecteurs nécessitant des sites adaptés. La question se pose alors de savoir ce que l'on peut montrer au public qui visite les laboratoires de physique des particules en France.

La pédagogie pour l'enseignement ou le grand public

On ne peut expliquer la physique subatomique au public en montrant des détecteurs en fonctionnement puisque les expériences ont lieu en dehors du laboratoire, souvent à l'étranger. Il faut donc trouver d'autres objets à montrer, des supports pour présenter la physique.

97 Le support le plus usité est bien sûr le poster, puisqu il permet d'expliquer la physique visuellement, avec un texte et des images, mais aussi de présenter des résultats expérimentaux, des graphiques, etc. Mais il est clair que le poster n'est pas suffisant. En physique subatomique, une grande partie des chercheurs travaillent, devant un écran d'ordinateur, à analyser la multitude de données fournies par les détecteurs de leur expérience. Cet aspect de la recherche, bien que fondamental, n'est malheureusement pas intéressant pour le public. Au cours des journées portes ouvertes, on a pu constater qu'il préfère qu'on lui montre directement des résultats, mais plus encore des détecteurs, que ce soit en fonctionnement ou même en construction.

Il faut donc réaliser des maquettes en trois dimensions des détecteurs et accélérateurs les plus représentatifs. La maquette de l'accélérateur TANDEM de l'Institut de physique nucléaire est en fonctionnement sur le campus d'Orsay; La maquette du futur détecteur CMS, réalisé par une collaboration internationale prendra des données d'ici 2006 auprès du futur collisionneur LHC (Large Hadron Collider) du CERN. La maquette est ici le seul moyen d'avoir une vue d'ensemble de ce détecteur cylindrique dont les dimensions finales seront gigantesques : 15 mètres de diamètre pour une longueur totale de près de 22 mètres! La maquette permet alors de présenter au public l'ensemble des caractéristiques de tous les sous-détecteurs et leur intérêt pour la physique étudiée.

Le troisième outil pédagogique est le prototype, rarement d'un détecteur complet mais plutôt d'un sous-détecteur et de son électronique, que les physiciens réalisent afin de valider l'ensemble des choix: matériaux, géométrie, ainsi que toutes les caractéristiques essentielles au bon fonctionnement comme l'efficacité ou la résolution des mesures effectuées par le futur détecteur. Lorsque cette étape est terminée, le prototype peut alors être utilisé comme objet démonstratif pour le public. Les prototypes ont en effet l'avantage d'être à l'échelle et d'avoir toutes les caractéristiques des vrais détecteurs, même s'ils ne participeront jamais aux expériences. Un prototype installé au LAL (Laboratoire de l'accélérateur linéaire) du calorimètre électromagnétique à argon liquide, qui est un sous-détecteur du futur détecteur ATLAS pour le LHC. Ce prototype représente environ $0,4 \%$ en volume du calorimètre et la 
même proportion en voies d'électronique. Au vue d'un schéma d'ATLAS, détecteur de 22 mètres de hauteur et 44 mètres de longueur (soit l'équivalent d'un immeuble de 5 étages!) et réalisé par 1750 physiciens et ingénieurs de 34 pays et 144 instituts, il apparaît que le calorimètre représente à peine $1 \%$ en volume total de détecteur, ce qui traduit bien l'intérêt $d$ 'avoir des prototypes de «taille humaine» pour expliquer le principe de fonctionnement du détecteur.

Il est aussi intéressant de présenter des petits détecteurs, ou des parties de détecteurs ayant cessé leur fonctionnement, accompagnés de posters explicatifs. Enfin, il arrive aussi que l'on crée des détecteurs uniquement dans un but pédagogique et pas pour une expérience: ils sont en ce cas parfaitement adaptés pour expliquer les phénomènes physiques.

101 Il est évident qu'il n'est pas possible d'avoir les maquettes de tous les détecteurs, et on ne trouve en général les prototypes des sous-détecteurs que dans les laboratoires participant à leur construction. Il faut donc s'intéresser au problème du déplacement des objets. Les présentations des posters et des détecteurs, que ce soit sous forme de maquettes, de prototypes, ou encore d'anciens détecteurs, doivent être pensées pour être itinérantes. C'était le cas de l'exposition pour les 25 ans de l'IN2P3 (Institut national de physique nucléaire et de physique des particules), qui utilisait l'ensemble des moyens pédagogiques à notre disposition et a circulé à travers toute la France entre 1996 et 1999. Pour faciliter les échanges, il serait certainement intéressant d'avoir une structure nationale, de type photothèque, pour l'échange des maquettes et prototypes. La sauvegarde des détecteurs

Depuis une dizaine d'années et grâce notamment à l'existence de structures comme Sciences ACO, il existe une prise de conscience collective dans notre domaine pour la sauvegarde du patrimoine instrumental. Mais les difficultés et les obstacles sont nombreux.

Le premier d'entre eux est naturellement le manque de locaux pour stocker ou présenter les détecteurs, problème lié à leurs grandes dimensions et au fait que nous manquons déjà de place pour stocker des objets en cours d'utilisation.

Le second est un problème de moyens, tant financiers qu'humains. En effet, il est nécessaire de disposer d'un budget adapté si l'on veut conserver des détecteurs ou des parties de détecteur: sans support pédagogique de type poster, et sans modification pour les rendre attractifs (par exemple, le remplacement de la matière des panneaux extérieurs d'une chambre à fils par du plastique pour que les fils soient visibles, ou encore la création d'un éclaté de détecteur pour montrer l'intérieur et les différentes parties, ce qui nécessite alors beaucoup plus de travail), les détecteurs par eux-mêmes ne peuvent être présentés au public. Et, bien sûr, les moyens humains sont aussi importants: il faut des personnes pour créer les posters associés au détecteur, et d'autres pour assurer le support technique, comme la gestion de l'ordinateur qui présente les données et résultats associés au détecteur.

En guise de conclusion

105 Dans le domaine de la physique subatomique, la taille des détecteurs est un handicap qui justifie l'utilisation massive de maquettes et de prototypes, même si nous conservons le plus possible de « petites " parties de détecteurs. Dans l'avenir et lors du démantèlement de son expérience, chaque physicien devrait se préoccuper au moins de 
la récupération des parties de sous-détecteurs réalisées dans son propre laboratoire. Peut-être faudrait-il y penser dès la réalisation du détecteur, pour prévoir les structures adéquates au moment où on dispose du potentiel technique nécessaire. Enfin, il me paraît évident que la sauvegarde du patrimoine instrumental est à la fois nécessaire, tant dans un but pédagogique que pour ne pas perdre les savoir-faire, mais aussi l'affaire de chacun. Cependant, elle ne pourra être réalisée que dans le cadre d'un effort national et concerté entre les disciplines et les laboratoires.

Conclusion de la conférence-débat $(\mathrm{H})$

Cette journée a montré l'intérêt d'un nombre de plus en plus important, mais encore trop faible, de chercheurs pour la sauvegarde du patrimoine scientifique de ces quelques dizaines d'années, ainsi que la nécessité de mettre en place une politique adaptée pour une sauvegarde d'urgence.

Il est avant tout important de souligner combien il est nécessaire de convaincre les scientifiques, qui vivent souvent dans l'immédiat, voire dans le futur, de l'importance de sauvegarder le patrimoine instrumental. En effet, des quantités de matériels scientifiques sont mises au rebut sans que les chercheurs n'aient l'idée de leur importance pour l'histoire des sciences et comme témoins d'une époque pour les générations à venir. C'est la construction brique par brique de l'édifice de la recherche qui, pour José Teixeira, masque au chercheur la perspective historique de l'importance des étapes scientifiques.

Mais que faut-il sauver?

Il ne suffit pas de conserver quelques objets, mais des expériences entières, en électrophysiologie par exemple, qui seules sont susceptibles de mener à une compréhension des processus de recherche de l'époque.

110 Ce réflexe de sauvegarde doit être complet, comprendre des dossiers et une mise en valeur immédiate, plutôt qu un stockage, intégrée à la politique du laboratoire concerné, comme cela est réalisé dans certaines universités étrangères prestigieuses : le cas de très gros laboratoires de physique nucléaire et des particules ou de TGE (très grands équipements), mais aussi de l'Institut d'optique, est donné en exemple.

Il est nécessaire de définir des critères de choix, de sélectionner les objets pertinents en fonction de l'importance qu' ils ont eue pour le progrès des sciences, à l'origine des « sauts » importants dans l'obtention de nouvelles connaissances pour la France et pour le monde.

112 L'organisation de cette sauvegarde doit reposer sur un réseau à un double niveau, local et national.

Un préalable nécessaire serait l'organisation d'une base de données qui permît la constitution de réseaux au niveau régional, voire national, pour que l'on connaisse ce qui existe, ce qui manque, ainsi que les localisations des divers exemplaires. Cette base de données devrait inclure les demandes des partenaires. Il va de soi que la création d'une telle base, et surtout sa gestion, pose des problèmes matériels qui ne pourront être résolus qu'avec la volonté des établissements de recherche.

$114 \mathrm{Au}$ niveau national, la première évidence est la nécessité de définir une politique de sauvegarde au sein des organismes de recherche. Par ailleurs, c'est la mission de quelques établissements (Muséum, Musée des arts et métiers, ainsi que le Palais de la 
Découverte et la Villette) de participer à ces opérations. C' est particulièrement celle du Musée des arts et métiers « de conserver et d'accroître le patrimoine national illustrant les progrès des sciences et des techniques ». Cela se réalise dans les domaines de l'instrumentation scientifique, des matériaux, de l'énergie, de la communication, des transports, de la mécanique, de la construction, et bientôt de l'instrumentation pour la santé. Le Musée des arts et métiers souhaite s'engager maintenant dans une campagne de sauvegarde avec des capacités de stockage nouvelles.

Il faut bien réaliser que, à ce niveau, une fois sélectionnés, les objets entrés dans les collections font partie du patrimoine national pour toujours. Les comités d'acquisition doivent assurer leur sélection avec beaucoup de discernement. Il est donc souhaitable d'avoir une sélection préalable afin d'enrichir des collections qui soient les plus complètes possible.

C'est au niveau local que ce type d'initiative est le plus adapté. Certains laboratoires, certaines universités, ont montré que ce réflexe de conservation et de sauvegarde est possible. Bien sûr, le nombre et l'ampleur de ces collections sont limités par les moyens à mettre en oeuvre pour leur conservation et leur exposition : locaux, personnels, coût de fonctionnement. Les collectivités locales commencent à s'intéresser à ce type d'action scientifique et technique. Pour les convaincre de la nécessité de sauvegarder ce patrimoine, il faut définir le but de cette démarche et la valorisation que l'on veut donner à ces collections.

$117 \mathrm{Au}$ cours de cette journée nous avons pris connaissance des actions menées au niveau d'un laboratoire, d'une délégation régionale. Différentes approches ont été proposées :

- simple exposition ;

- confrontation autour d'un objet pour montrer la science en train de se faire ;

- explication de la science par des maquettes et des prototypes (pour les TGE);

- parcours dans les laboratoires.

118 Malheureusement, cette approche simple et "objective» est de moins en moins adaptée, et plusieurs expériences exposées pendant cette journée ont montré qu'elles doivent être conçues dans un objectif d'intégration dans la culture de notre société.

Enet, les chercheurs ne peuvent, avec leurs appareils, répondre seuls aux questions

telles que se les pose le public. La science n' a pas fait cet effort d'intégration avec la société depuis plus d'un siècle. Depuis Pasteur, le système scientifique, dominé par la compétition et l'accumulation rapide de nouveaux résultats, s'est coupé de celle-ci. La société par ses questions sur de grands sujets d'actualités (énergie, biotechnologies...) l'oblige à reconsidérer ces liens.

Une médiation devient de plus en plus souvent nécessaire. Diverses actions, en particulier celles des Centres de culture scientifique, technique et industrielle (CCSTI d'Orléans, de Nantes...), ont montré comment ils pouvaient, avec les chercheurs, faire comprendre au grand public à quoi sert la science, 1 intéresser à son déroulement, à son évolution par des témoignages, des films, des expositions.

121 C'est dans une démarche globale que les laboratoires doivent s'impliquer, avec les chercheurs, avec les médiateurs afin qu'ils puissent parler à l'Homme et non pas enseigner. Il est important de travailler en favorisant l'interdisciplinarité. Il faut 
profiter de «l'individualité » des laboratoires pour montrer le caractère global de la recherche, l'importance d'intégrer les diverses connaissances accumulées pour avoir une vision cohérente de la réalité, indépendamment de l'entrée disciplinaire, qu elle soit physique, chimique ou biologique. Bref, il s'agit de faire redécouvrir la science au public, de restituer au travail de recherche son caractère $d$ 'aventure intellectuelle avec sa rigueur, le respect de l'expérience, de la mesure.

Ce n'est que par cet effort d'échange avec la société, avec l'Homme, de présence sensible à tous, que la culture scientifique et technique pourra être reconnue comme une véritable culture. C' est certainement par un plus grand dialogue avec les sciences humaines et sociales que cette réflexion pourra aboutir.

\section{BIBLIOGRAPHIE}

Bibliographie

[1] R. Bimbot, J. Dumarchez, J. Laberrigue, C. Nicault, H. Ostrowiecki, J.-P. Repellin et C. Victor, « Rapport d'étape du groupe de réflexion sur la sauvegarde du patrimoine de l'IN2P3 », octobre 2000, non publié.

[2] G. Frick, La Physique nucléaire à Strasbourg, Saisons d'Alsace 106, 1989.

[3] C. Nicault, Le patrimoine de l'IN2P3 - «État des travaux, rapport du Comité pour l'histoire du CNRS », août 2000, non publié.

[4] R. Bimbot, ALICE ou l'essor des ions lourds, film $16 \mathrm{~mm}, 26 \mathrm{~min}$, réalisé par D. Garabédian et S. Guyon, ENS Production et IPN Orsay, 1989. Existe aussi en version longue (52 min) et en version anglaise (26 $\mathrm{min})$.

[5] R. Bimbot et M. Paty, « Vingt-cinq années d'évolution de la physique nucléaire et des particules ", in 25 ans de recherche â l'IN2P3, édité par J. Yoccoz, Frontières, Paris, 1996.

[6] Exposition réalisée par R. Bimbot et G. Edelheit (1993).

\section{NOTES}

1.On enregistre en fait un signal oscillatoire dont la fréquence nominale varie en fonction de la valeur absolue du signal mesuré (en plus ou en moins). Comme la variation de fréquence est limitée à environ $40 \%$ de la valeur nominale, il faut que celle-ci soit élevée, imposant une grande vitesse de défilement de la bande $(38 \mathrm{~cm} / \mathrm{s}$, voire $76 \mathrm{~cm} / \mathrm{s}$ ) pour enregistrer les fréquences élevées composant le signal étudié. 2.Un grand détecteur de physique des particules peut atteindre les dimensions d'un immeuble de six étages. 
3.« Étude sur le patrimoine scientifique : les enjeux culturels de la mémoire scientifique », Catherine Roth, novembre 2000 (http://www.cnrs.fr/Archives/ARISC/ rapports/rapports.html).

\section{RÉSUMÉS}

\section{LA SAUVEGARDE DU PATRIMOINE INSTRUMENTAL}

Conférence-débat du 6 juin 2001, Orsay

Ouverture par Raymond Duval, délégué régional du CNRS Ile-de-France Sud et introduction de la journée par André Kaspi, président du Comité pour l'histoire du CNRS.

Que conserver et comment?

A - L'instrumentation scientifique, un élément essentiel du patrimoine, José Teixeira, Laboratoire Léon Brillouin, CNRS-CEA

B - La disparition des matériels et du savoir-faire en neurosciences, Jacques Stinnakre, Laboratoire de neurobiologie cellulaire et moléculaire, CNRS, Gif-sur-Yvette

C - La sauvegarde des petits et grands instruments en physique nucléaire et en physique des particules, René Bimbot, Institut de physique nucléaire d'Orsay, IN2P3, CNRS, université Paris-Sud

D. Historique et préservation du développement instrumental à l'Institut d'optique, Pierre Chavel, Laboratoire Charles Fabry de l'Institut d'optique d'Orsay

Comment valoriser le patrimoine conservé ?

E - Le patrimoine scientifique: un exemple de valorisation et de collaboration entre un organisme de recherche et un CCSTI, Danièle Le Roscoüet-Zelwer, chargée de communication de la Délégation du CNRS Centre-Auvergne-Limousin

F - L'Anneau de collisions d'Orsay (ACO) : un exercice de muséographie, Pierre Dhez, Laboratoire de spectroscopie atomique et ionique (LSAI) et d'utilisation du rayonnement électromagnétique (LURE) et Pierre Marin, Laboratoire de l'accélérateur linéaire d'Orsay

G - L'intérêt des maquettes et prototypes en physique des particules, Corinne Augier, Laboratoire de l'accélérateur linéaire d'Orsay

H - Conclusion par Daniel Thoulouze, directeur de la culture scientifique et technique et du Musée des arts et métiers

\section{INDEX}

Mots-clés : Patrimoine instrumental 